\title{
MEGARA anti-reflective coatings: theoretical and observed throughput estimations
}

Ortiz, R., Carrasco, E., Páez, G., Pompa, O., SánchezBlanco, E., et al.

R. Ortiz, E. Carrasco, G. Páez, O. Pompa, E. Sánchez-Blanco, A. Gil de Paz, J. Gallego, J. Iglesias-Páramo, "MEGARA anti-reflective coatings: theoretical and observed throughput estimations," Proc. SPIE 10706, Advances in Optical and Mechanical Technologies for Telescopes and Instrumentation III, 107065H (10 July 2018); doi: 10.1117/12.2313976

Event: SPIE Astronomical Telescopes + Instrumentation, 2018, Austin, Texas, United States 


\title{
MEGARA anti-reflective coatings: theoretical and observed throughput estimations
}

\author{
R. Ortiz ${ }^{\text {a }}$, E. Carrasco ${ }^{\text {a }}$, G. Páez ${ }^{\text {b }}$, O. Pompa ${ }^{\text {b }}$, E. Sánchez-Blanco ${ }^{\text {c }}$, A. Gil de Paz ${ }^{\text {d }}$ J. Gallego ${ }^{\text {d }}$ J. \\ Iglesias-Páramo $^{\mathrm{e}}$ \\ ${ }^{a}$ Instituto Nacional de Astrofísica Óptica y Electrónica, Luis Enrique Erro 1, Tonantzintla, Puebla, \\ Mexico; ${ }^{b}$ Centro de Investigaciones en Óptica, León, Guanajuato, Mexico; ${ }^{\mathrm{c}}$ Fractal SNLE, Spain; \\ ${ }^{\mathrm{d}}$ Universidad Complutense de Madrid, Spain; ${ }^{\mathrm{e}}$ Instituto de Astrofísica de Andalucía, Spain.
}

\begin{abstract}
MEGARA is the new integral field unit (IFU) and multi-object (MOS) spectrograph successfully commissioned at Gran Telescopio Canarias, in August 2017. MEGARA provides spectral resolutions R (fwhm) 6000, 12000 and 20000, via volume phase holographic gratings, at very high efficiency in both IFU and MOS modes. In the case of MEGARA main optics and pupil elements optics, the surfaces in contact with air have an anti-reflective (AR) coatings to minimize the Fresnel losses at the interface glass-air. In this work we present the designs and calculation of the total throughput of the optical system based in the transmission measurements of the AR coated witness samples. The results reflect the benefits of having implemented customized AR coatings for the mean angle of incidence on each surface as the measured throughput was better than the requirements. We analyze the effects of the pupil elements AR coatings for each spectral configuration.
\end{abstract}

Keywords: antireflection coatings, optical design, thin films, optical testing, broadband coatings, MEGARA, GTC

\section{INTRODUCTION}

MEGARA $^{1,2,3,4}$ (Multi-Espectrógrafo en GTC de Alta Resolución para Astronomía) is an optical Integral-Field Unit (IFU) and Multi-Object Spectrograph (MOS) designed for the GTC 10.4m telescope, in operation in La Palma (Spain). It has been developed under a contract between the Universidad Complutense de Madrid (UCM) and GRANTECAN. The MEGARA Consortium is led by UCM and has as co-partners the Instituto Nacional de Astrofísica, Óptica y Electrónica (INAOE. Mexico), the Instituto de Astrofísica de Andalucía (IAA-CSIC, Spain) and the Universidad Politécnica de Madrid (UPM, Spain).

The instrument has an IFUs plus a MOS mode: a large compact bundle covering $12.5 \operatorname{arcsec} \times 11.3 \operatorname{arcsec}$ on sky with $100 \mu \mathrm{m}$ fiber-core; a fiber MOS positioner that allows to place up to 100 mini-bundles, 7 fibers each, with $100 \mu \mathrm{m}$ fibercore, within a 3.5 arcmin x 3.5 arcmin field of view, around the IFU. The fibers, organized in bundles, end in the pseudoslit plate located at the entrance of MEGARA spectrograph. The collimator and camera optics and optomechanics have been completed and delivered. The full instrument was installed on site during August 2017, all the spectral configurations, in the IFU and MOS modes are available and fulfill the design specifications.

MEGARA optical system design consists of:

- a pseudo-slit, which has $119 \mathrm{~mm}$ of length, and is composed by an arrangement of optical fibers. It is curved on a sphere surface of a radii of curvature (ROC) of $1075 \mathrm{~mm}$;

- the collimator, which is composed by one single and two double lenses, its focal length is $484.4 \mathrm{~mm}$ (at $632.8 \mathrm{~nm}$ ) and its f-number is 3.03 . The optical elements of the collimator are presented in table 2 . The only aspheric is COLL-S1;

- 18 high performance VPHs gratings, which main characteristics are shown in Table 1;

- the camera, composed by two doublets and three singlets with a total focal length of $245.9 \mathrm{~mm}$ (average paraxial value) and f-number of 1.54. The last lens is the cryostat window and the image field is $61.4 \mathrm{~mm} \times 61.4 \mathrm{~mm}$;

- $\quad$ and the $4 \mathrm{k} \times$ 4k pixel EV CCD detector. 
The shutter and the cut-order filters are placed in the collimator barrel. Detailed information of the design requirements and as-built characteristics of the spectrograph are available in other publications $s^{4,5,6,7,8}$.

Figure 1 shows the component distribution of MEGARA spectrograph along with the materials and diameters of each lens.

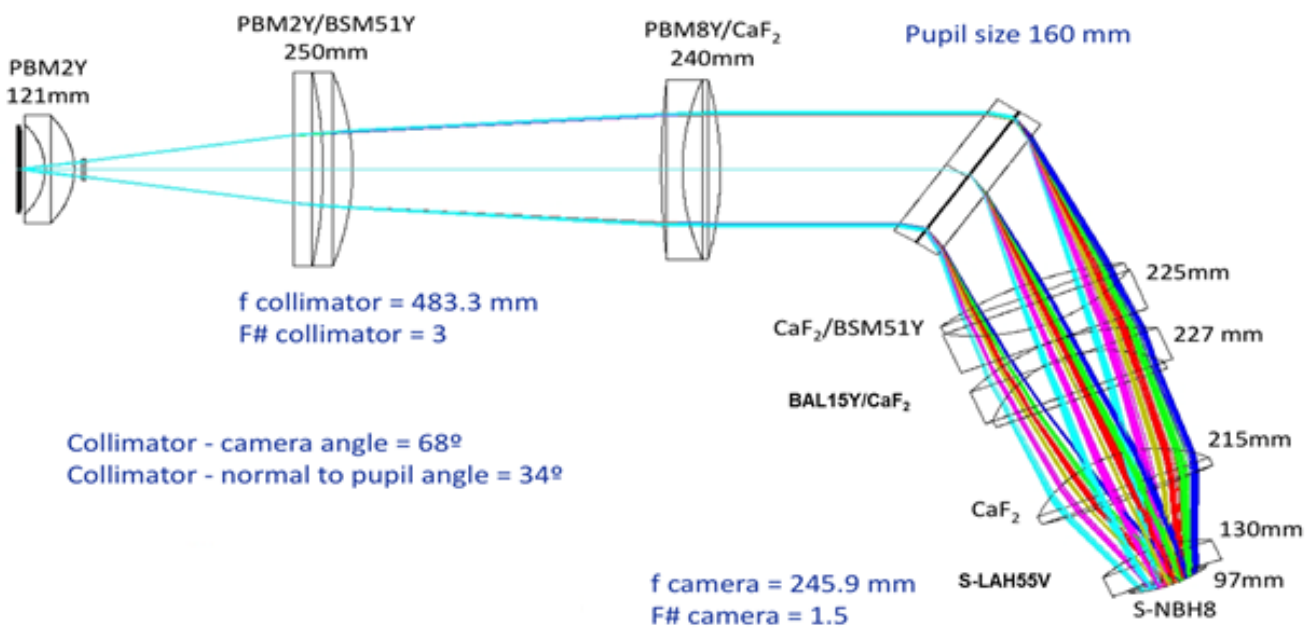

Figure 1. MEGARA optic system layout.

MEGARA initial requirements were resolutions of RFWHM $\sim 6,000,12,000$ and 18,700, respectively for the low-, midand high-resolution Volume Phase Holographic (VPH) gratings. The complete list of MEGARA gratings requirements ${ }^{1}$ is given in table 1 .

As a high-level requirement, it was defined that the total MEGARA throughput, considering fiber link and spectrograph, should be better than $30 \%$ at the central wavelength of the VPH675-LR i.e. low-resolution grating working in the red; $\sim 670 \mathrm{~nm}$. This requirement was for the instrument only i.e. without including the GTC .

\begin{tabular}{|c|c|c|c|c|c|c|c|}
\hline VPH NAME & SETUP & $\bar{~} \overline{\mathbf{R}_{\text {FWHM }}}$ & $\begin{array}{c}\lambda_{1}-\lambda_{2} \\
\AA\end{array}$ & $\begin{array}{l}\lambda \mathbf{c} \\
\AA\end{array}$ & $\begin{array}{c}\Delta \lambda(@ \lambda c) \\
\AA\end{array}$ & $\begin{array}{c}\begin{array}{c}\Delta \mathbf{v} \\
\mathrm{km} / \mathrm{s}\end{array} \\
\end{array}$ & $\begin{array}{c}\text { lin res } \\
\AA / \text { pix }\end{array}$ \\
\hline VPH405-LR & LR-U & 6028 & $3653-4386$ & 4051 & 0.672 & 50 & 0.17 \\
\hline VPH480-LR & LR-B & 6059 & $4332-5196$ & 4800 & 0.792 & 49 & 0.20 \\
\hline VPH570-LR & LR-V & 6080 & $5143-6164$ & 5695 & 0.937 & 49 & 0.23 \\
\hline VPH675-LR & LR-R & 6099 & $6094-7300$ & 6747 & 1.106 & 49 & 0.28 \\
\hline VPH799-LR & LR-I & 6110 & $7220-8646$ & 7991 & 1.308 & 49 & 0.33 \\
\hline VPH890-LR & LR-Z & 6117 & $8043-9630$ & 8900 & 1.455 & 49 & 0.36 \\
\hline VPH410-MR & MR-U & 12602 & $3917-4277$ & 4104 & 0.326 & 24 & 0.08 \\
\hline VPH443-MR & MR-UB & 12370 & $4225-4621$ & 4431 & 0.358 & 24 & 0.09 \\
\hline VPH481-MR & MR-B & 12178 & $4586-5024$ & 4814 & 0.395 & 25 & 0.10 \\
\hline VPH521-MR & MR-G & 12035 & $4963-5443$ & 5213 & 0.433 & 25 & 0.11 \\
\hline VPH567-MR & MR-V & 11916 & $5393-5919$ & 5667 & 0.476 & 25 & 0.11 \\
\hline VPH617-MR & MR-VR & 11825 & $5869-6447$ & 6170 & 0.522 & 25 & 0.13 \\
\hline VPH656-MR & MR-R & 11768 & $6241-6859$ & 6563 & 0.558 & 25 & 0.14 \\
\hline VPH712-MR & MR-RI & 11707 & $6764-7437$ & 7115 & 0.608 & 26 & 0.15 \\
\hline VPH777-MR & MR-I & 11654 & $7382-8120$ & 7767 & 0.666 & 26 & 0.17 \\
\hline VPH926-MR & MR-Z & 11638 & $8800-9686$ & 9262 & 0.796 & 26 & 0.20 \\
\hline VPH665-HR & HR-R & 18700 & $6445-6837$ & 6646 & 0.355 & 16 & 0.09 \\
\hline VPH863-HR & HR-I & 18701 & $8372-8882$ & 8634 & 0.462 & 16 & 0.12 \\
\hline
\end{tabular}

Table 1. MEGARA Baseline gratings. 18 configurations for several spectral ranges. 
Scientific measurements to be made with MEGARA define the transmission specifications. The main optics required broadband AR coatings in the wavelength range from 370 to $980 \mathrm{~nm}$ with $\mathrm{R}<1.3 \%$. The AR coatings have been optimized for a different mean AOI for each surface. An overview ${ }^{12}$ has been made on the whole system and the requirements for each main optics element.

MEGARA pupil elements wavelength range transmission is defined by the different spectral configurations: six for LR, ten for MR and two for HR. The specification for these elements was $\mathrm{R}<0.4 \%$ with the goal of $\mathrm{R}<0.3 \%$ for the mean AOI that is different for each subgroup (LR, MR and HR). Table 2 provides a summary of all the pupil elements and their specific AOI and spectral range.

In this work we present the final AR coatings of the complete optical components of the spectrograph, main and pupil elements. Along that, a comparison between the theoretical throughput and the real throughput is shown,

\section{Windows and Prisms AR Coatings}

\begin{tabular}{|c|c|c|c|c|}
\hline Element & Substrate & Surface AR Width & Mean AOI (deg) & $\mathbf{R}(\%)$ \\
\hline $\begin{array}{c}\text { (LR-U) } \\
\text { windows (2) }\end{array}$ & \multirow{6}{*}{$\begin{array}{c}\text { Fused Silica } \\
(\mathrm{SK} 1300) \\
\mathrm{ne}=1.46058\end{array}$} & $360 \mathrm{~nm}-445 \mathrm{~nm}$ & \multirow[t]{6}{*}{34} & \multirow[t]{14}{*}{$\mathrm{R}<0.3 \%$} \\
\hline $\begin{array}{c}\text { (LR-B) } \\
\text { windows (2) }\end{array}$ & & $390 \mathrm{~nm}-570 \mathrm{~nm}$ & & \\
\hline $\begin{array}{c}\text { (LR-V) } \\
\text { windows (2) }\end{array}$ & & $470 \mathrm{~nm}-620 \mathrm{~nm}$ & & \\
\hline $\begin{array}{c}\text { (LR-R) } \\
\text { windows (2) }\end{array}$ & & $550 \mathrm{~nm}-780 \mathrm{~nm}$ & & \\
\hline $\begin{array}{c}(\text { LR-I) } \\
\text { windows (2) }\end{array}$ & & $670 \mathrm{~nm}-920 \mathrm{~nm}$ & & \\
\hline $\begin{array}{c}(\mathrm{LR}-\mathrm{Z}) \\
\text { windows (2) }\end{array}$ & & $750 \mathrm{~nm}-990 \mathrm{~nm}$ & & \\
\hline $\begin{array}{c}\text { (MR-U) } \\
\text { prisms (2) }\end{array}$ & \multirow{8}{*}{$\begin{array}{c}\text { PMB2Y } \\
\mathrm{nd}=1.62004\end{array}$} & $380 \mathrm{~nm}-440 \mathrm{~nm}$ & \multirow[t]{8}{*}{10.5} & \\
\hline $\begin{array}{l}\text { (MR-UB) } \\
\text { prisms (2) }\end{array}$ & & $410 \mathrm{~nm}-480 \mathrm{~nm}$ & & \\
\hline $\begin{array}{l}\text { (MR-B) } \\
\text { prisms (2) }\end{array}$ & & $440 \mathrm{~nm}-520 \mathrm{~nm}$ & & \\
\hline $\begin{array}{c}\text { (MR-G) } \\
\text { prisms (2) }\end{array}$ & & $480 \mathrm{~nm}-560 \mathrm{~nm}$ & & \\
\hline $\begin{array}{c}(\mathrm{MR}-\mathrm{V}) \\
\text { prisms (2) }\end{array}$ & & $520 \mathrm{~nm}-610 \mathrm{~nm}$ & & \\
\hline $\begin{array}{l}\text { (MR-VR) } \\
\text { prisms (2) }\end{array}$ & & $570 \mathrm{~nm}-660 \mathrm{~nm}$ & & \\
\hline $\begin{array}{l}\text { (MR-R) } \\
\text { prisms (2) }\end{array}$ & & $610 \mathrm{~nm}-700 \mathrm{~nm}$ & & \\
\hline $\begin{array}{l}\text { (MR-RI) } \\
\text { prisms (2) }\end{array}$ & & $660 \mathrm{~nm}-760 \mathrm{~nm}$ & & \\
\hline
\end{tabular}




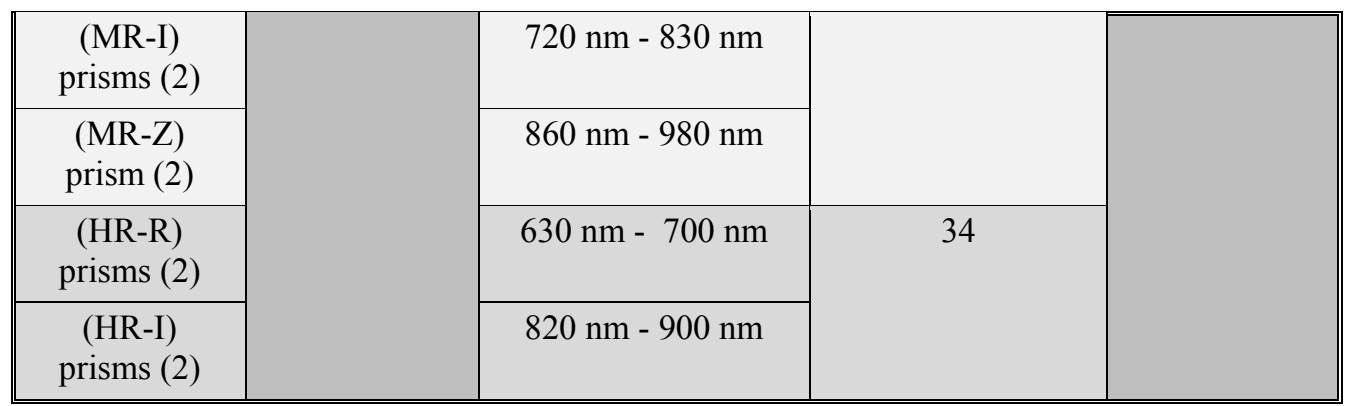

Table 2. Summary of the pupil elements AR coatings designed for MEGARA.

\section{ANTIREFLECTIVE COATINGS DESIGN}

When light passes through any surface some part of it will be reflected, other refracted or absorbed, this is well known as Snell's Law. On uncoated glass substrates, approximately $4 \%$ will be reflected if it is a Crown glass and it can be as large as $10 \%$ in Flint glass at each interface. This degrades the total transmission in the range from $80 \%$ to $92 \%$. Losses start to become unbearable in optical systems over two elements, as they also translate in possible ghost images. In order to minimize this loss of light, thin film coatings are deposited on the substrate through different techniques, the most popular being the so called physical vapor deposition (PVD), to enhance the inherent transmission through interference effects.

The properties of this type of coating are dependent on the refractive index of the substrate, the refractive index of the thin film, the thickness of the thin film, the angle of incidence of the light and the wavelength. Each coating is designed to obtain a relative phase shift of $\pi$ between the reflected beams in the boundaries of the film, if this condition is met then destructive interference occurs. This can be translated to $\frac{\lambda}{4}$ in terms of wavelength, thus the coating will have to be an odd number of $\frac{\lambda}{4}$ in thickness for the desired wavelength.

Regarding the refractive index needed for the coating, it can be shown that if

$$
R=\left(\frac{n_{0}-n_{s}}{n_{0}+n_{s}}\right)^{2}
$$

then,

$$
n_{f}=\sqrt{n_{0} n_{s}}
$$

where

$R$ is the reflectance

$n_{f}$ is the index of refraction of the coating

$n_{0}$ is the index of refraction of air

$n_{s}$ is the index of refraction of the substrate

Most of the times this refractive index cannot be achieved by currently available materials, instead a stack of different materials with different refractive indexes is used. This means that antireflection coatings can range from a simple single layer having virtually zero reflectance at just one wavelength, to a multilayer system of more than a dozen layers, having virtually zero reflectance over a range of several octaves.

When the design of these coatings is done to perform on normal incidence there can be some problems at greater AOI, and the antireflection capabilities are degraded. The accumulated phase in the layer relative to the phase of the incident light decreases as the AOI increases. This can be resolved by noting that the ray will exit the layer spatially offset from where it entered and will interfere with reflections from incoming rays that had to travel further (thus accumulating more phase of their own) to arrive at the interface. The net effect is that the relative phase is actually reduced, shifting the 
coating, such that the anti-reflection band of the coating tends to move to shorter wavelengths as the optic is tilted. Another effect of non-normal incidence angles is for the coating to be polarization-dependent.

In the case of the main optics of MEGARA a wide spectral region (370nm to $980 \mathrm{~nm}$ ) needed to be covered, thus the apparent dispersion of the equivalent periods represented a problem. This dispersion was reduced by using equivalent periods of one-eighth-wave thickness instead of quarter-wave. Each quarter-wave in the original design is then replaced by two periods in series. This led us to eight layer coatings. ${ }^{8}$

In astronomy, AR coatings are important especially in the blue region where several optical materials have high reflection and/or absorption. These coatings are often analyzed together with the dispersive element and CCD efficiency, which by default has an AR coating on it. They play a key role in the final throughput of a refractive instrument, such as MEGARA.

The MEGARA main optics AR designed coatings consist in eight layers of three different materials to fulfill the requirements of transmission in the wavelength range at the AOI presented in table 3.

The range of the AR for the pupil elements is considerably narrower compared to that of the main optics, thus the requirements are more demanding. To fulfill them, several four layer designs were produced and optimized for each spectral band presented in table 4 .

Theoretical designs were achieved through different methods ${ }^{9,10}$ based on the materials available for the deposition. Then an optimization of the design was implemented defining the targets of reflection and the mean AOI of each optical element.

A Damped Least-Square algorithm was used for optimization with iterations variating from 100 to 200. Specialized software was used and due to the wide range of wavelengths in the main optics, dispersion effects of the thin film materials and the substrate are considered.

A total of 18 designs were developed for the main optics and another 18 for the pupil elements including LR windows and MR - HR prisms.

\section{FINAL DEPOSITION RESULTS OF THE PUPIL ELEMENTS}

After the fabrication of each of the optical elements, the deposition of the coating materials was made in the Integrity 39 Denton Vacuum Deposition System located at CIO, León, Guanajuato and the transmission of the coating on a witness was measured in an Agilent Cary-5000-UV-Vis-NIR® spectrophotometer.

Several witness samples of each substrate were polished and coated to make the transmission measurements prior and during the final deposition. Three transmission measurements were carried for each one and the average was computed. To extract the transmission of the coated surface of the witness from the measurement, the procedure was the following:

1. A simulation of the substrates was made, in Zemax, emulating a witness with an ideally perfect AR where the transmission is $99.99 \%$.

2. Theoretical values of the total transmission are obtained.

3. Fresnel losses on the uncoated surface of the sample and absorption losses of the substrate from the measurement were eliminated dividing by the theoretical transmission of the witness sample.

In cases when the $\mathrm{AOI} \geq 10^{\circ}$ the measurement could not be taken directly at the angle specified. To sort out this limitation, the measurements of transmission of the witnesses were taken at normal incidence and then extrapolated to the designated AOI of each element using the method described below:

i. Theoretical transmission functions of the coating at normal incidence and at the mean AOI were produced aided by optical software.

ii. A coefficient function was achieved by dividing the transmission function at the mean AOI by that at normal incidence.

iii. The transmission of the witness sample was measured at normal incidence using the Cary-5000 spectrophotometer at CIO. 
iv. The witness sample transmission at the mean AOI was inferred from the experimental data measured at normal incidence, applying the coefficients obtained in (ii).

v. The effects of absorption and the reflections in the uncoated side of the sample were eliminated using the method described above, steps 1- 3 .

In figure 2, we present the performance results of the final coated elements of the main optics which can be reviewed in detail $^{12}$.

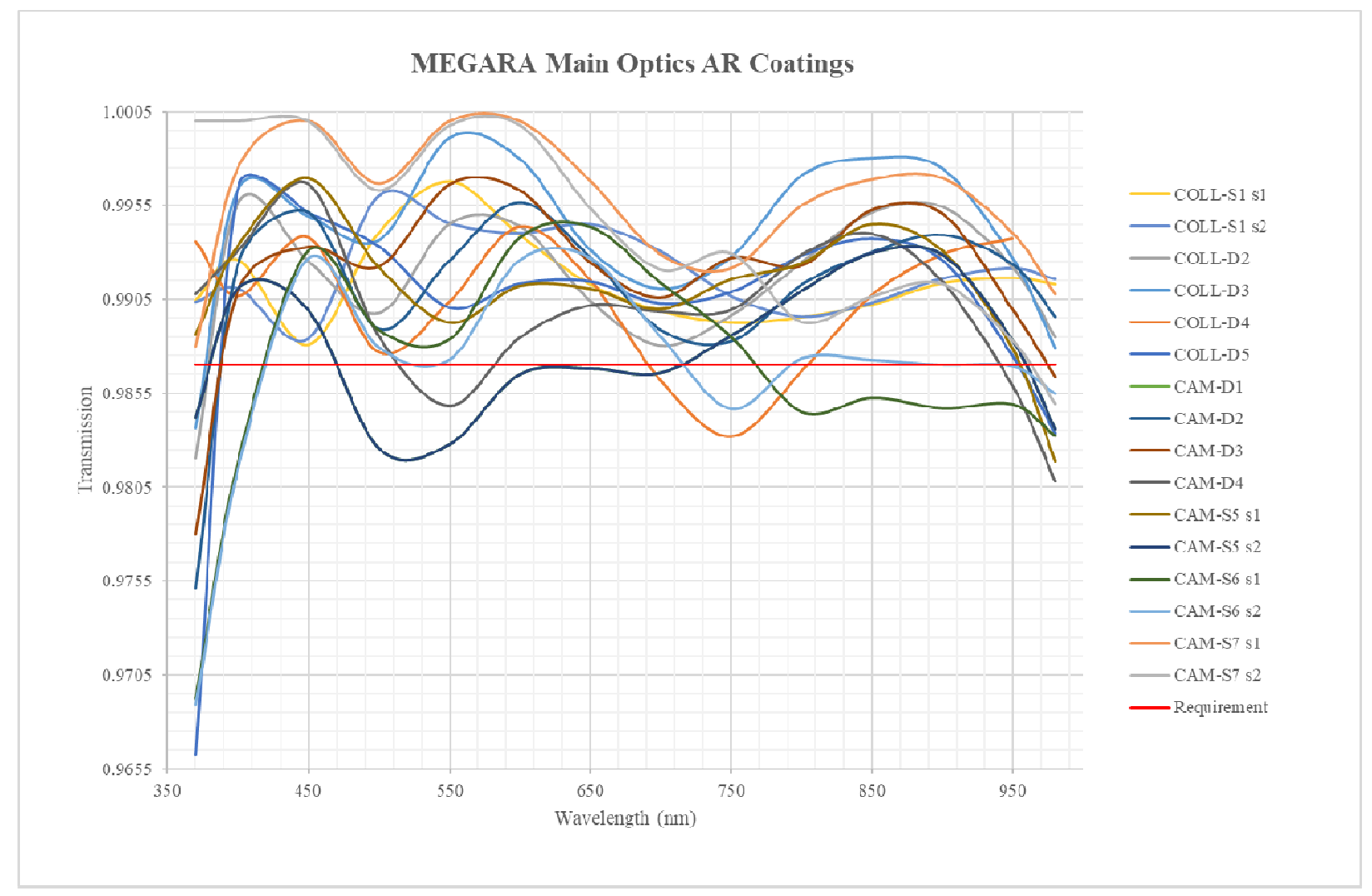

Figure 2. Measured surface transmission of the main optics final coated elements. 


\begin{tabular}{l}
\hline \hline Coated Element \\
\begin{tabular}{|l|l|}
\hline Field Lens & 0.011 \\
\hline COLL-S1 S1* & 0.013 \\
\hline COLL-S1 S2* & 0.013 \\
\hline COLL-D2 & 0.009 \\
\hline COLL-D3 & 0.006 \\
\hline COLL-D4 & 0.009 \\
\hline CAM-D2 & 0.009 \\
\hline CAM-D3 & 0.008 \\
\hline CAM-S5 S1 & 0.009 \\
\hline \hline CAM-S5 S2 & 0.009 \\
\hline CAM-S6 S1 & 0.012 \\
\hline CAM-S6 S2 & 0.013 \\
\hline CAM-S7 S1 & 0.004 \\
\hline CAM S7 S2 & 0.007 \\
\hline AVERAGE TOTAL LOSS & 0.124 \\
\hline
\end{tabular}
\end{tabular}

Table 3. Average reflectance of main optics surfaces coated elements.

The results for the pupil elements final experimental transmission are presented in figures 3, 4 and 5. For the LR windows the requirement of average reflectance $\leq 0.003$ is not completely fulfilled because it was the most demanding. For the Fused Silica LR windows, the specification was for a mean AOI was $34^{\circ}$ in $85 \mathrm{~nm}$ to $250 \mathrm{~nm}$ wide wavelength intervals. In comparison for the PBM2Y MR prisms the mean AOI was $10.5^{\circ}$ for $70 \mathrm{~nm}$ to $120 \mathrm{~nm}$ wide wavelength intervals. For the PBM2Y HR prisms the specification was also very demanding as the mean AOI was $34^{\circ}$. See Table 2, Thus, the decision was made to go on with these depositions as they do not affect substantially the overall performance of the spectrograph.

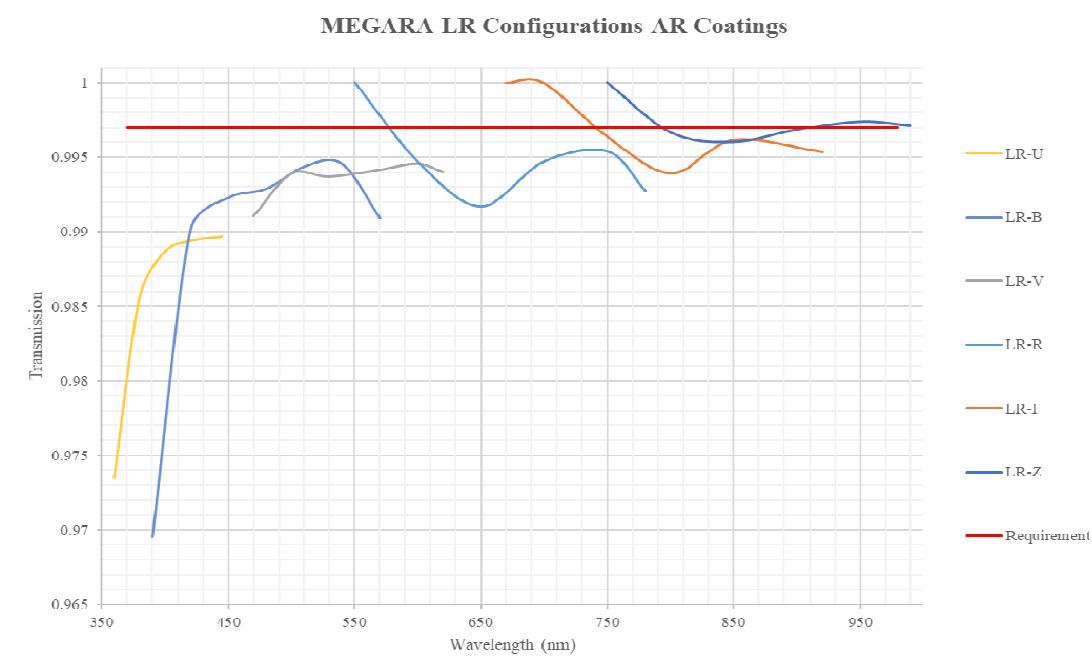

Figure 3.Measured transmission of the LR coated windows 


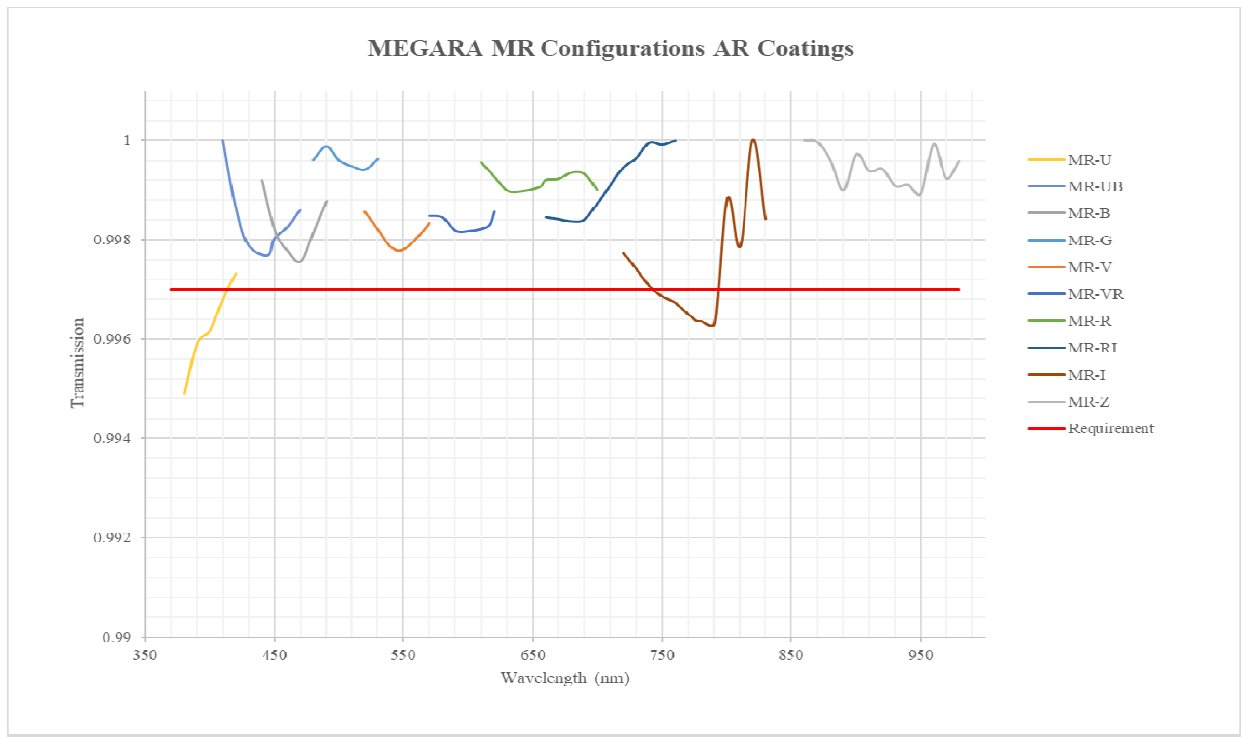

Figure 4. Measured transmission of the coated MR prisms.

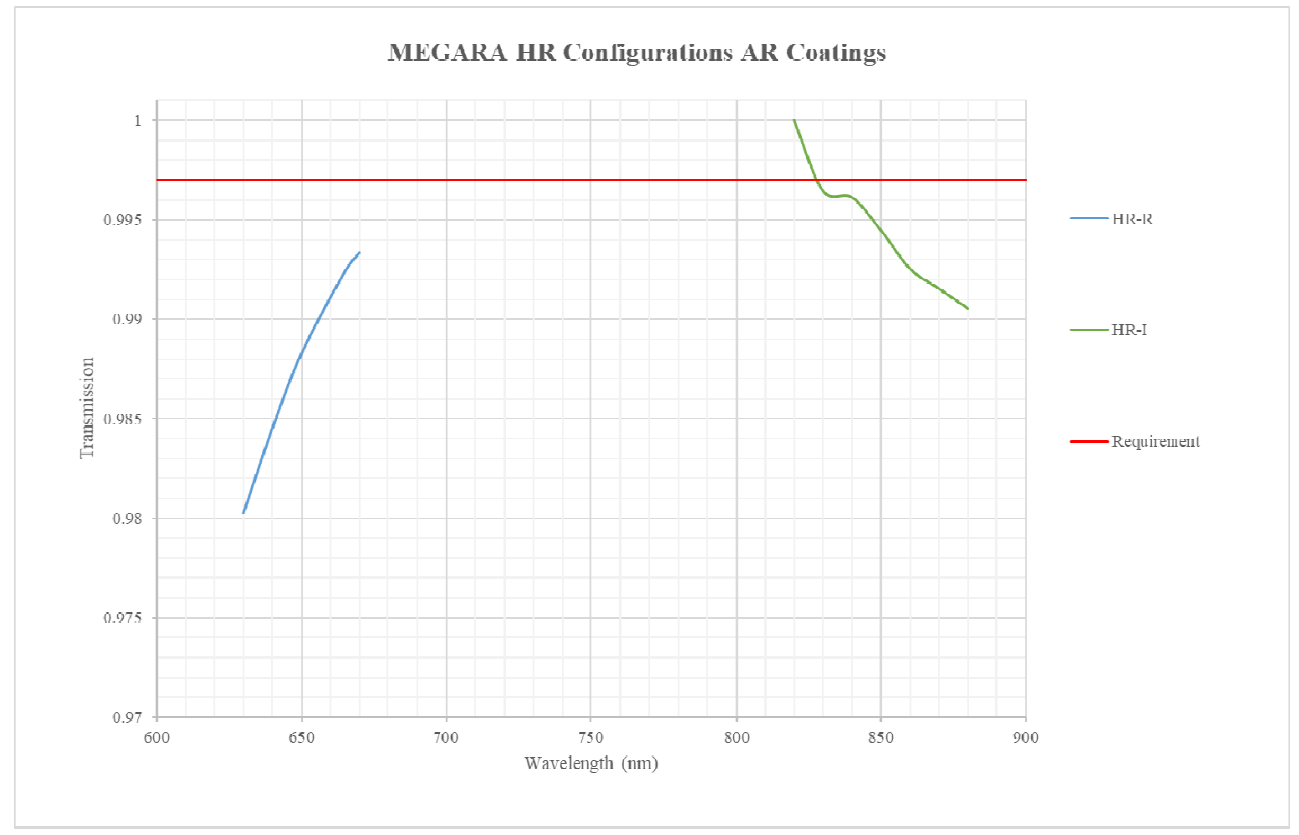

Figure 5. Measured transmission of the HR coated prisms surfaces.

\begin{tabular}{|c|c|c|c|c|c|}
\hline $\begin{array}{c}\text { Low } \\
\text { Resolution }\end{array}$ & $\begin{array}{c}\text { Average } \\
\text { Reflectance }\end{array}$ & $\begin{array}{c}\text { Medium } \\
\text { Resolution }\end{array}$ & Ave R & $\begin{array}{c}\text { High } \\
\text { Resolution }\end{array}$ & Ave $R$ \\
\hline LR-U & 0.005 & MR-U & 0.003 & HR-R & 0.009 \\
\hline LR-B & 0.009 & MR-UB & 0.002 & HR-I & 0.006 \\
\hline
\end{tabular}




\begin{tabular}{|c|c|c|c|c|c||}
\hline LR-V & 0.004 & MR-B & 0.002 & - & - \\
\hline LR-R & 0.004 & MR-G & 0.001 & - & - \\
\hline LR-I & 0.003 & MR-V & 0.002 & - & - \\
\hline LR-Z & 0.003 & MR-VR & 0.002 & - & - \\
\hline- & - & MR-R & 0.001 & - & - \\
\hline- & - & MR-RI & 0.001 & - & - \\
\hline- & - & MR-I & 0.003 & - & - \\
\hline- & - & MR-Z & 0.001 & - & \\
\hline
\end{tabular}

Table 4. Average reflectance of LR, MR and HR coated pupil elements.

\section{THROUGHPUT ESTIMATION}

Throughput requirements are essential in any optical system, especially for astronomy. These are the considerations used for the throughput budget as we have divided the contributors to the GTC + MEGARA Throughput budget in the following parts:

- GTC optical mirror (reflectivity as in GTC documentation).

- MEGARA Folded-Cassegrain subsystems, which include all MEGARA subsystems from Field lens to the fiber exit at the spectrograph entrance.

- MEGARA spectrograph common optical path subsystems, which include the collimator plus camera optics spectrograph and the detector.

- $\quad$ MEGARA gratings, which provides the values for all Low-Resolution (LR), Medium-Resolution (MR) and High-Resolution (HR) gratings.

MEGARA spectrograph main optical path

\begin{tabular}{|c|c|c|c|c|c||}
\hline$\lambda(\mathbf{n m})$ & $\begin{array}{l}\text { Main Optics Initial } \\
\text { Estimation }\end{array}$ & $\begin{array}{l}\text { Main Optics AR } \\
\text { Design Estimation }\end{array}$ & $\begin{array}{l}\text { Main Optics } \\
\text { Measurements }\end{array}$ & Detector & Main optical path \\
\hline 365 & 0.126 & 0.320 & 0.393 & 0.650 & 0.255 \\
\hline 370 & 0.184 & 0.355 & 0.408 & 0.700 & 0.285 \\
\hline 380 & 0.317 & 0.475 & 0.430 & 0.800 & 0.344 \\
\hline 390 & 0.430 & 0.625 & 0.527 & 0.850 & 0.448 \\
\hline 405 & 0.534 & 0.712 & 0.673 & 0.910 & 0.612 \\
\hline 410 & 0.556 & 0.730 & 0.679 & 0.920 & 0.624 \\
\hline 440 & 0.637 & 0.766 & 0.715 & 0.910 & 0.650 \\
\hline
\end{tabular}




\begin{tabular}{|c|c|c|c|c|c|}
\hline 480 & 0.695 & 0.773 & 0.723 & 0.900 & 0.650 \\
\hline 500 & 0.721 & 0.772 & 0.727 & 0.890 & 0.647 \\
\hline 520 & 0.732 & 0.776 & 0.740 & 0.880 & 0.651 \\
\hline 550 & 0.749 & 0.788 & 0.758 & 0.890 & 0.674 \\
\hline 570 & 0.749 & 0.791 & 0.767 & 0.890 & 0.682 \\
\hline 600 & 0.749 & 0.808 & 0.779 & 0.900 & 0.701 \\
\hline 620 & 0.750 & 0.805 & 0.770 & 0.910 & 0.700 \\
\hline 665 & 0.751 & 0.793 & 0.750 & 0.920 & 0.690 \\
\hline 675 & 0.751 & 0.783 & 0.745 & 0.925 & 0.689 \\
\hline 700 & 0.752 & 0.775 & 0.734 & 0.930 & 0.682 \\
\hline 715 & 0.753 & 0.772 & 0.734 & 0.930 & 0.682 \\
\hline 750 & 0.755 & 0.773 & 0.736 & 0.930 & 0.684 \\
\hline 775 & 0.755 & 0.797 & 0.755 & 0.900 & 0.679 \\
\hline 800 & 0.755 & 0.823 & 0.775 & 0.880 & 0.682 \\
\hline 825 & 0.755 & 0.835 & 0.784 & 0.850 & 0.667 \\
\hline 850 & 0.755 & 0.840 & 0.794 & 0.800 & 0.635 \\
\hline 865 & 0.754 & 0.843 & 0.794 & 0.720 & 0.572 \\
\hline 900 & 0.750 & 0.834 & 0.795 & 0.600 & 0.477 \\
\hline 925 & 0.747 & 0.812 & 0.767 & 0.575 & 0.441 \\
\hline 940 & 0.746 & 0.784 & 0.750 & 0.400 & 0.300 \\
\hline 980 & 0.742 & 0.735 & 0.706 & 0.200 & 0.141 \\
\hline
\end{tabular}

Table 5. MEGARA Spectrograph main optical path throughput budget. We include the contributions from main optics and detector.

Table 5 summarizes the throughput expectations for the spectrograph common optical path only. This estimation includes the following contributors:

- Main Optics, which is composed by the collimator and the camera. We have assumed $1.5 \%$ Fresnel losses per glass/air interfaces and this implies that this is a worse case. INAOE and CIO provided coatings better than $1.3 \%$.

- Detector includes the Quantum efficiency of the CCD231-E74 (Astro multi-2 AR coating) as provided by e2v.

The column main optical path summarizes the spectrograph transmission taken into account the experimental values of transmission of the optical components and the CCD contributions. 


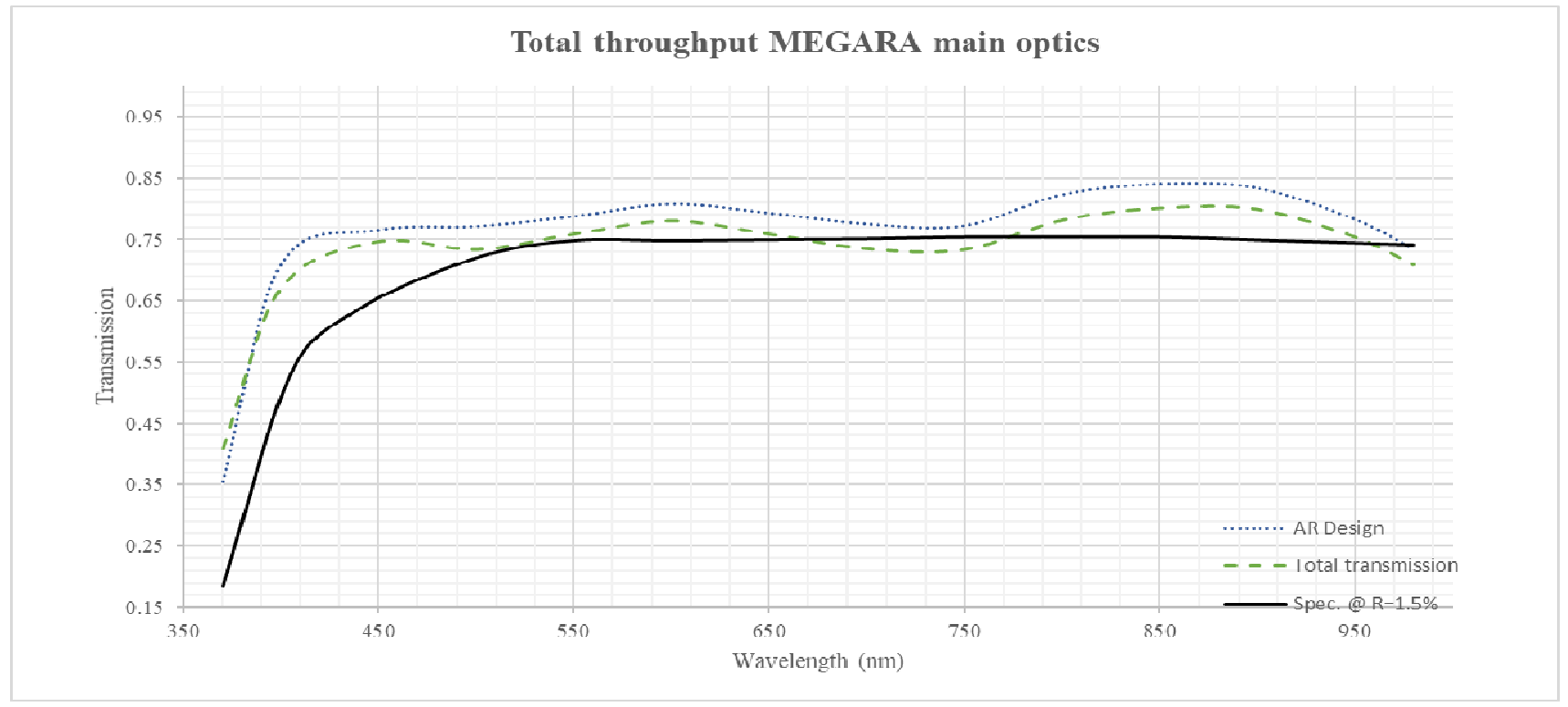

Figure 6. Main optical path overall transmission without detector. Plotted are the initial specs, the expected transmission with AR designs and experimental transmission curve.

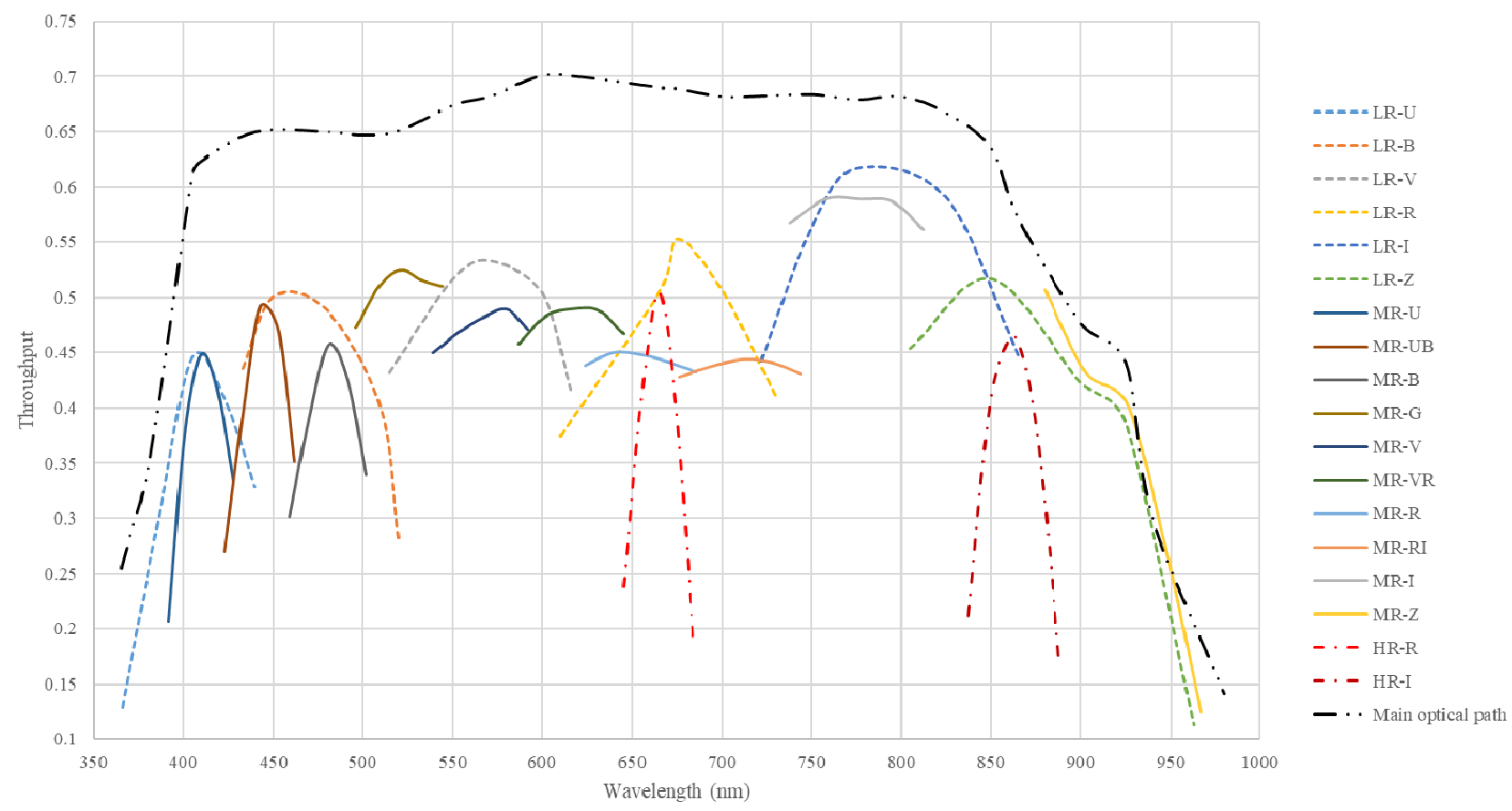

Figure 7. Transmission of the spectrograph in its different configurations without considering the GTC or the fiber link. The black curve is the throughput of the main optical path considering the detector. The individual curves correspond the VPH measured transmission reported by WASATCH or MEGARA team together with the contributions of the coated surfaces of the pupil elements.

In figure 7, the experimental values of the main optics and the pupil elements are presented as the spectrograph throughput, without considering GTC and fiber link contributions. The pupil elements AR coatings are taken into account along with the VPH efficiency values calculated by MEGARA team ${ }^{13}$.

If we consider the whole GTC optical path plus the fiber bundles the average transmission values of approximately 0.5 for the main optics, 0.9 for the detector in the blue wavelength, and 0.8 in the red part of the spectrum. Additionally, if 
we consider pupil elements to have $0.79,0.67$ and 0.76 for the LR, MR and HR configurations. We obtain theoretical efficiencies close to 0.33 in the blue $(400 \mathrm{~nm})$, and 0.44 in the red $(850 \mathrm{~nm})$. These values are among the best efficiencies currently seen in optical spectrographs with astronomical applications; this makes MEGARA a unique instrument in terms of total transmission.

\section{CONCLUSIONS}

The results of experimental measurements of optical transmission of the whole spectrograph optics have been studied and compiled. This work make emphasis in the AR coatings of the main optics and the pupil elements, the throughput estimations are better than initially estimated.

All the main optic elements from the collimator and the camera, the 36 VPHs windows and the 24 prisms were manufactured, coated and delivered to UCM in 2015 and 2016. MEGARA was integrated and tested at UCM during 2016. MEGARA was delivered to the observatory in March 2017. The integration at GTC was carried out from March to April 2017. The daytime commissioning was during May and June 2017 and the nighttime commissioning in July and August 2017. The observations proved that MEGARA fulfills are the requirements in both modes, LCB and MOS, in the 18 spectral configurations. MEGARA is a case of success as it was delivered in time, within budget and fulfilling all the requirements. MEGARA has been offered to the community for the 2018B semester. The transmission of MEGARA is one of the bests in the current generation of optical spectrographs in a $10 \mathrm{~m}$ class telescope.

INAOE and CIO have worked together to develop state of the art AR coatings. This has expanded their expertise in this field and are looking forward to collaborate in future astronomical projects that have very demanding requirements in overall transmission.

\section{REFERENCES}

[1] Gil de Paz, A., et al., "MEGARA: the future optical IFU and multi-object spectrograph for the $10.4 \mathrm{~m}$ GTC telescope", Proc. of SPIE Vol. 8446, 84464Q-1-9 (2012)

[2] Gil de Paz, A., et al., "MEGARA: a new generation optical spectrograph for GTC", Proc. of SPIE Vol. 9147, 23 (2014)

[3] Gil de Paz, A., et al., "MEGARA, the new intermediate-resolution optical IFU and MOS for GTC: getting ready for the telescope", Proc. of SPIE, this volume (2016)

[4] Carrasco, E., et al. "MEGARA, the R=6000-20000 IFU and MOS GTC" Proc. SPIE 10702, Paper No. 10702-42 (2018)

[5] Carrasco, E., et al., "Performance of MEGARA spectrograph optical elements", Proc. of SPIE, this volume (2016)

[6] Carrasco, E., et al., "MEGARA spectrograph optics", Proc. of SPIE, Vol. 8011, 80112D (2012)

[7] Sánchez-Blanco, E., García-Vargas, M. L., Ortiz, R., "MEGARA Detailed Design: Spectrograph Optics", TEC/MEG/100, MEGARA consortium internal technical report, 1-59 (2014)

[8] Ortiz, R., et al., "Inverse analysis method to optimize the optic tolerances of MEGARA: the future IFU and MultiObject Spectrograph for GTC", Proc. of SPIE, Vol. 9147, id. 914749 (2014)

[9] Macleod, H. A., [Thin-Film Optical Filters], CRC Press, Florida, 4th Edition (2010).

[10] Schallenberg, U., "Design principles for broadband AR coatings", Proceedings of the Society of Photo-Optical Instrumentation Engineers 7101 710103-1-710103-8 (2008)

[11] Schallenberg U., "Antireflection design concepts with equivalent layers" Appl Opt 45 1507-1514 (2006)

[12] R. Ortiz, et al., "Design and testing of AR coatings for MEGARA optics", Proc. SPIE 9908, Ground-based and Airborne Instrumentation for Astronomy VI, 990886 (2016)

[13] I. Martínez-Delgado, et al., "MEGARA: large pupil element tests and performance", Proc. SPIE 9912, Advances in Optical and Mechanical Technologies for Telescopes and Instrumentation II, 99124D (2016) 